\title{
Noninvasive cardiovascular findings in familial amyloid polyneuropathy
}

\author{
TOSHITAMI SAWAYAMA, TERUYUKI KURIHARA, AND SHUKURO ARAKI \\ From the Department of Internal Medicine, Kawasaki Medical School, Kurashiki, Fapan and \\ The Third Department of Internal Medicine, Medical College of Miyazaki, Miyazaki, Fapan
}

SUMMARY The cardiovascular system was examined in 19 cases of familial amyloid polyneuropathy. In a group of patients with neurological involvement, various cardiac abnormalities were common, $\overrightarrow{\hat{N}}$ including orthostatic hypotension, prominent apex cardiographic A waves, abnormal apical systolic $\infty_{\infty}^{\infty}$ waves (bulges), systolic murmurs, mid-systolic clicks, QS waves, atrioventricular block, left bundle-을 branch block, and abnormalities of ejection time and pre-ejection period. Though there was one case with pronounced cardiac abnormality despite a normal neurological state, and though cardiovascular $z$ symptoms appeared later than neurological symptoms, the degree of cardiac involvement generally paralleled the severity of the neurological disorder.

Familial amyloid polyneuropathy initially involves the peripheral and autonomic nervous systems and later affects the cardiovascular system. Little is known about the cardiovascular functional effects of the disease, apart from the electrocardiographic abnormalities, and there has been no precise comparison between cardiac and neurological findings in this disease. Therefore, we studied several families with this disorder in Arao City, Kumamoto, where there is a very large incidence of this illness, in order to elucidate the type and severity of associated cardiac dysfunction using noninvasive methods.

Received for publication 15 September 1977

\section{Subjects}

We studied 12 cases, 7 men and 5 women, of familial amyloid polyneuropathy with neurological symptoms (the affected group), and 7 cases, 2 men and 5 women, without neurological symptoms (the non-affected group) (Table 1).

The average age of the affected group was $34 \cdot 3 \vec{\circ}$ years. The duration of the disease ranged from 13 month to 17 years. On the basis of results of peripheral nerve function tests (measurements of motor? and sensory nerve conduction velocities of ulnar, median, and peroneal nerves using Medelec MS6-1@ Electromyograph) there were 7 cases showing no response to electric stimuli or a significant delay in 3 .

Table 1 Peripheral nerve function tests in 12 patients with abnormal neurological findings (the affected group)

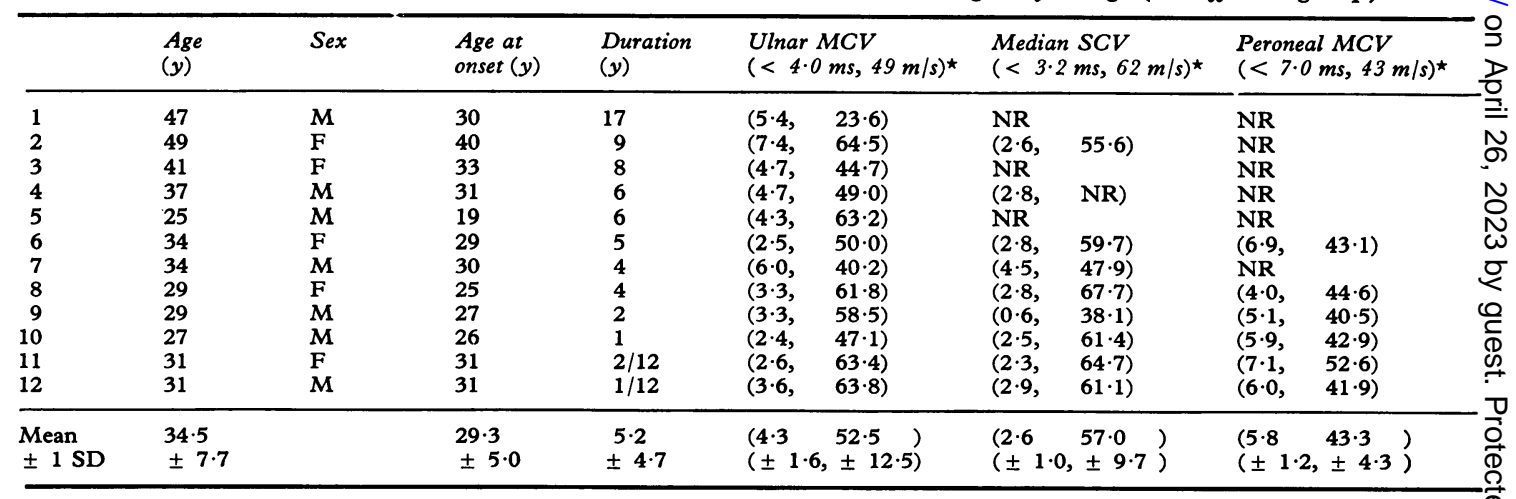

MCV, motor conduction velocity; SCV, sensory conduction velocity; * normal values (latency, minimum velocity); $\mathrm{NR}$, no response to the nerve stimulations. 
Table 2 Cardiovascular symptoms and physical signs in 2 groups of patients with familial amyloid polyneuropathy (FAP)

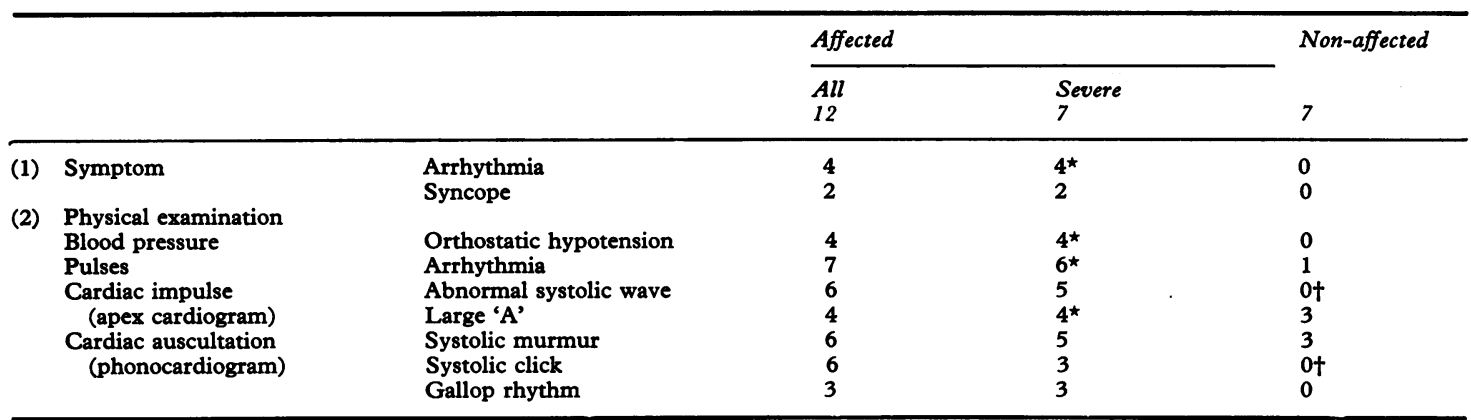

* Statistically significant difference between the severe and mild subgroups.

+ Statistically significant difference between the affected and the non-affected groups.

nerve conduction velocity. These cases were designated the severe group.

The average age of the non-affected group was 33.9 years.

\section{Method}

Each subject underwent the following: (1) careful historical review of symptoms related to the cardiovascular system, (2) physical examination of the cardiovascular system, (3) standard 12-lead electrocardiogram, (4) polygraphic recordings by our standard method (Sawayama et al., 1973), using simultaneous phonocardiogram, apex cardiogram, carotid arterial pulse, and jugular venous pulse. In

Table 3 Electrocardiogram and polygraphic recordings in 2 groups of patients with familial amyloid polyneuropathy

\begin{tabular}{llll}
\hline & Affected & $\begin{array}{l}\text { Non- } \\
\text { affected }\end{array}$ \\
\cline { 2 - 3 } All & Severe & 7 \\
12 & 7 & 7 \\
\hline
\end{tabular}

(3) Electrocardiogram QS pattern Sinoatrial block

AV block complete incomplete

BB block (left)

Extrasystole

ST-T change

Low voltage (limb)

Left ventricular hypertrophy

(4) Left ventricular systolic

time intervals

Abnormal ET and PEP $\quad 7 \quad 5 \quad 0+$

AV, atrioventricular; BB, bundle-branch; ET, ejection time; PEP, pre-ejection period.

* Statistically significant difference between severe and mild subgroups.

† Statistically significant difference between the affected and the non-affected groups. addition, measurements were made of the left ventricular systolic time intervals (LVSTI) [total systolic phase (Q-II), ejection time (ET), preejection period (PEP) and ET/PEP ratiol (Sawayama et al., 1973). The blood pressure was measured in the supine position and after 3 minutes in the sitting position.

\section{Results}

Tables 2 and 3 list the results of all observations, and provide indications of statistical significance.

In the affected group the duration of subjective cardiovascular symptoms was 0 to 4 years, which was significantly shorter than that of the neurological symptoms. The main cardiovascular abnormalities were arrhythmias, syncopal attacks, and orthostatic hypotension. The apex cardiogram disclosed abnormal systolic waves (bulges) and prominent A wave, while the phonocardiogram showed significant systolic murmurs, mid-systolic clicks, and gallop rhythms. Such abnormalities were especially obvious in the neurologically severe group.

In contrast, in the non-affected group, arrhythmias, prominent $\mathrm{A}$ wave, and systolic murmurs were noted only in the patients with diabetes mellitus and hypertension.

Fig. 1 shows one patient from the affected group with an abnormal systolic bulge and mid-systolic click.

The electrocardiograms of the affected group showed infarction-like QS patterns, sinoatrial blocks, atrioventricular blocks, and left bundlebranch blocks. These abnormalities were more frequent in the neurologically severe group (Table 3). Significant prolongations of pre-ejection period (PEP) and significant reductions of ET/PEP were also more predominant in the severe group. 

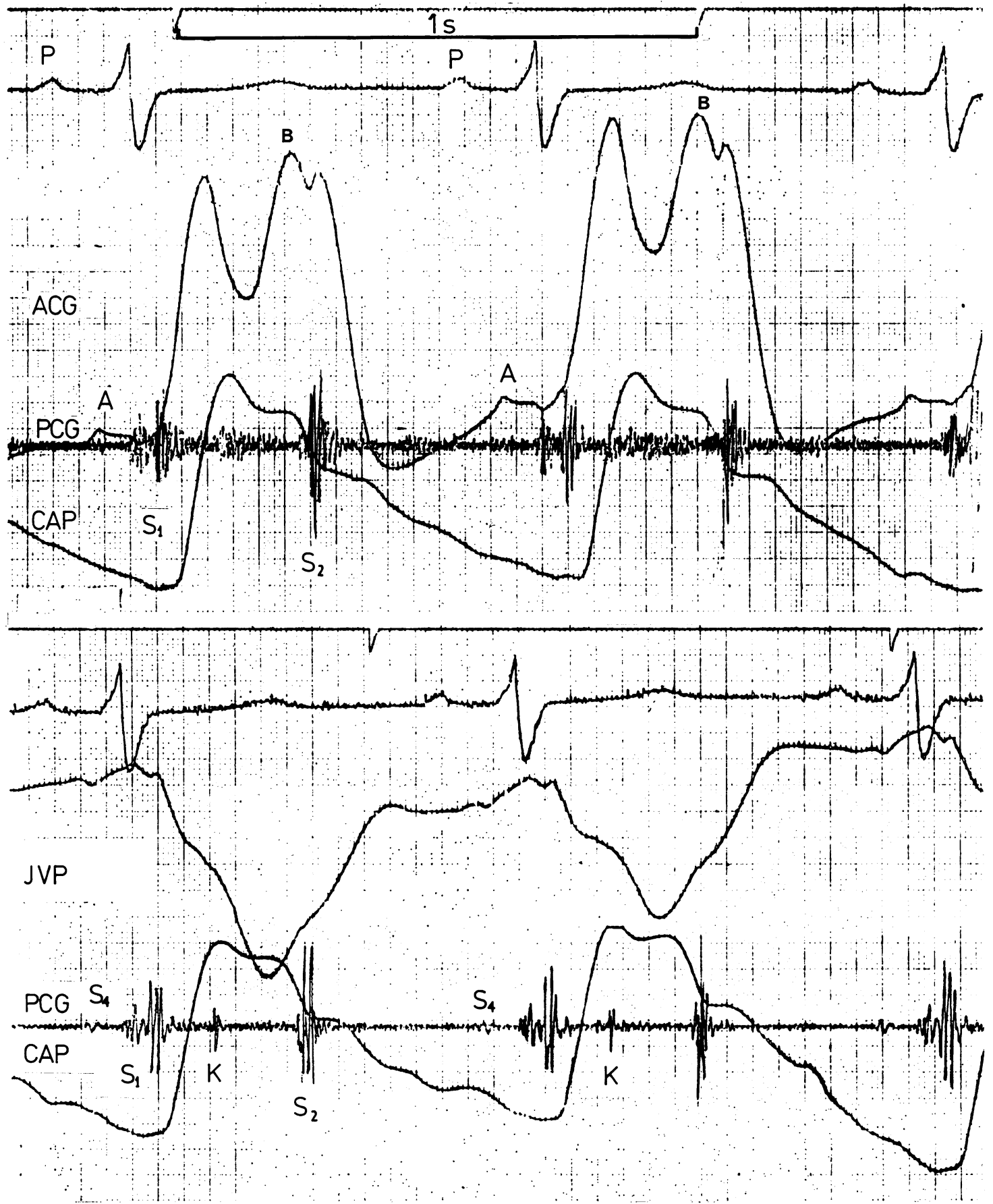

Fig. 1 Case 1 in the affected group shows an abnormal systolic wave with late systolic bulge $(B)$ in the apex cardiogram and a mid-systolic click $(K)$ on phonocardiogram. ACG, apex cardiogram; $C A P$, carotid arterial pulse; fVP, jugular venous pulse; $P C G$, phonocardiogram. 


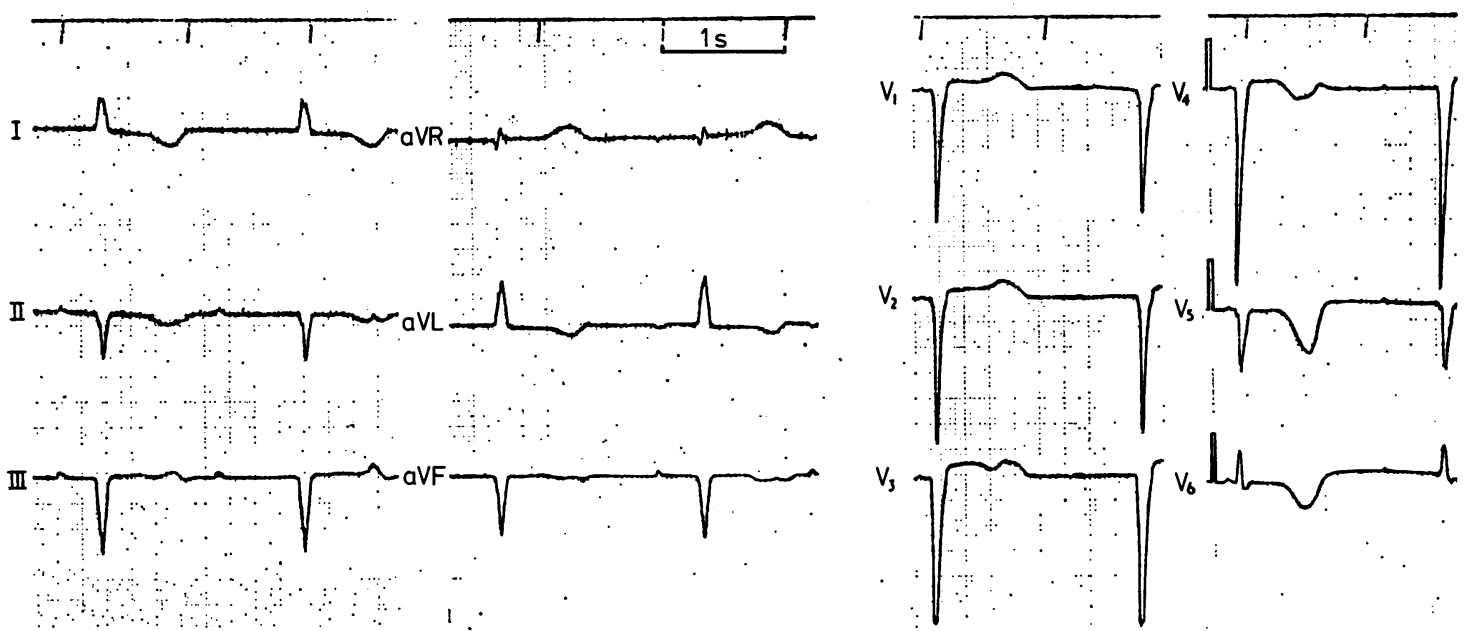

Fig. 2 Case 2 in the affected group shows complete atrioventricular block, a QS pattern in II, III, aVF, V1-5, left axis deviation, left bundle-branch block, and distinct $S T$-T changes.

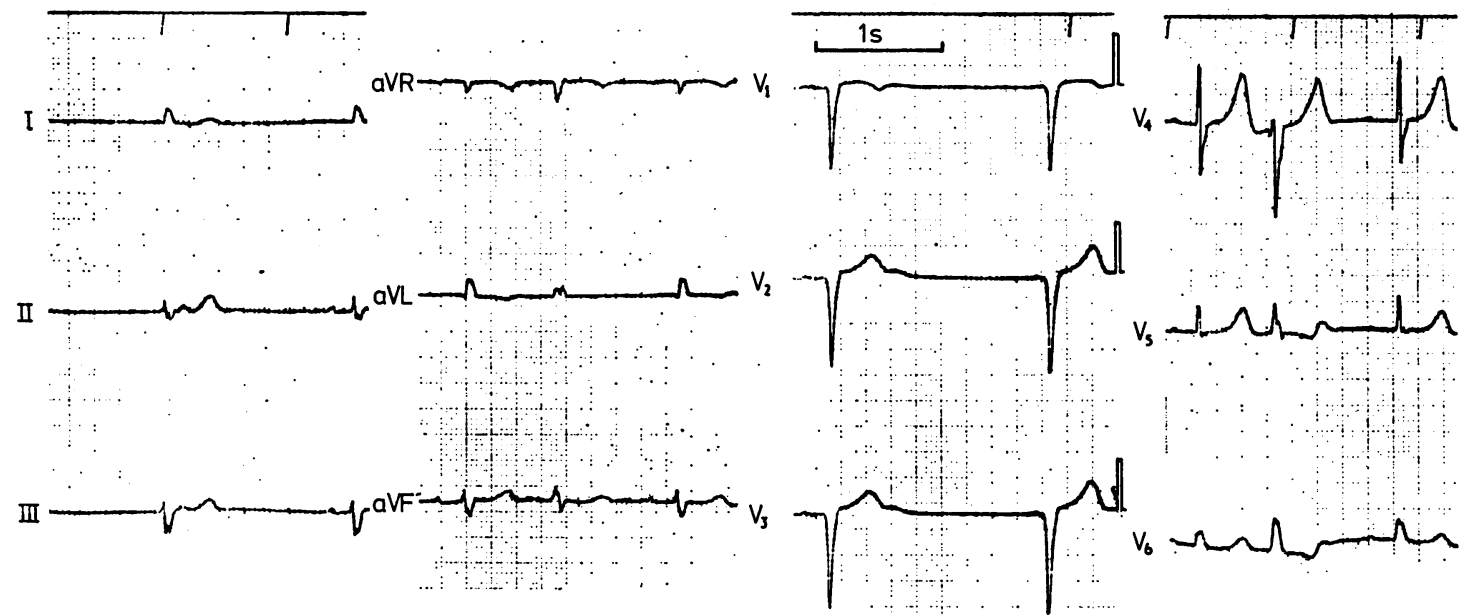

Fig. 3 Case 3 in the affected group reveals sinoatrial block, a ventricular premature beat, a QS pattern in V1-3, left axis deviation, and left bundle-branch block.

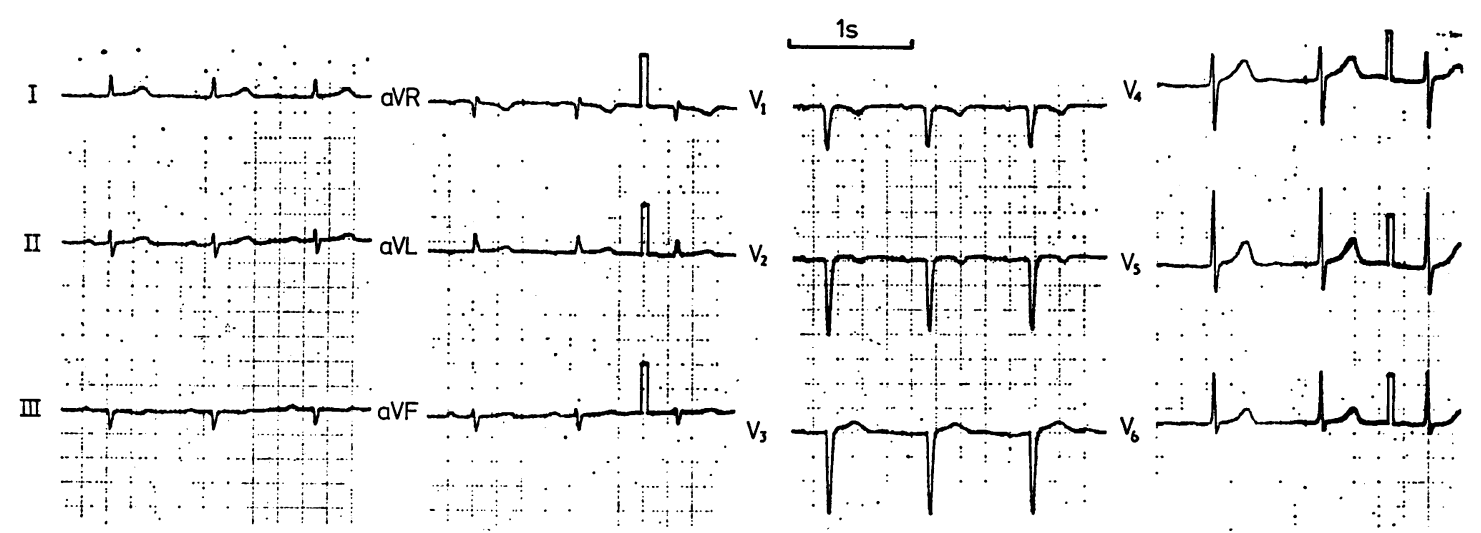

Fig. 4 Case 5 in the non-affected group shows first degree atrioventricular block, low voltage in limb leads, left axis deviation, and a QS pattern in VI-3. 
The electrocardiograms of the non-affected group (with the exception of those cases having diabetes mellitus and hypertension) were abnormal in only one individual. In the non-affected group the left ventricular systolic time interval was normal.

Representative electrocardiographic abnormalities are illustrated in Figs 2 to 4.

\section{Discussion}

Cardiac involvement has been variably emphasised in previous reports of familial amyloid polyneuropathy. Frederiksen et al. (1962) found that congestive heart failure was often present when neurological symptoms were minimal. In the 3 cases reported by Allenworth et al. (1969), atrial standstill was noted but heart failure was not evident. Recently, Coelho and Pimentel (1961) in Portugal reported 34 cases of familial amyloid neuropathy of a special type. These cases showed distinct neuropathy but no cardiac symptoms. There were no pronounced changes in heart sounds, and all patients were in sinus rhythm. Only 20 of the cases showed abnormalities in the electrocardiogram, including premature beats, right and left ventricular hypertrophy, incomplete atrioventricular block, bundle-branch block, and non-specific $T$ wave abnormalities. In the 4 cases with familial neuropathy reported by Buja et al. (1970), 1 had heart failure while 3 showed low voltage in the electrocardiogram.

These and other previous reports have suggested that various familial groups with amyloidosis may present quite different clinical syndromes, depending on the predilection for amyloid deposition in the nervous, cardiovascular, or renal systems, or in the conduction system vs. the general musculature of the heart. A similarity has been reported, in this regard, between the cases with familial amyloid neuropathy in Portugal and those in Japan (Andrade et al., 1970). However, in the Japanese cases, the degree of cardiac dysfunction closely paralleled the severity of the neurological disorder, and a wide variety of electrocardiographic abnormalities were encountered, including infarction patterns, sinoatrial and atrioventricular conduction disturbances, left bundle-branch block, tachyarrhythmias, and non-specific ST-T abnormalities. Two of the patients with conduction disturbances ( 1 sinoatrial and 1 atrioventricular block) required permanent pacemakers because of syncopal attacks. Orthostatic hypotension was associated with severe cardiac dysfunction in 4 of our cases, though Buja et al. (1970) have pointed out that this relation is not consistent.

We have not found a full report of polygraphic findings in familial amyloid neuropathy. In our cases, an abnormal systolic wave was found in the apex cardiogram in 6 of 12 cases of the affected group (in 5 of the 7 severe cases). This abnormality has previously been reported predominantly in patients with ischaemic heart disease and cardiomyopathy (Benchimol, 1977). A prominent systolic murmur was recorded in 4 phonocardiograms and a mid-systolic click in 6 tracings. These findings may be related to papillary muscle dysfunction (Barlow et al., 1968) because it is pertinent that half of the patients with extensive amyloid deposits in papillary muscles at necropsy had apical systolic murmurs (Buja et al., 1970).

The left ventricular systolic time interval was abnormal in 8 of the 12 cases of our affected group. These abnormalities may have been, partly, the result of conduction abnormalities, but they strongly suggested primary myocardial involvement in view of their usually close relation to ventricular performance (Weissler et al., 1968), and the frequently abnormal values found in other types of cardiomyopathy (Benchimol, 1977).

We wish to thank Dr Allan V. N. Goodyer, Professor of Medicine, Yale University School of Medicine, and $\mathrm{Mr}$ Seiichi Yamamoto in our laboratory, for their assistance.

\section{References}

Allenworth, D. C., Rice, G. J., and Lowe, G. W. (1969). Persistent atrial standstill in a family with myocardial disease. American fournal of Medicine, 47, 775-784.

Andrade, A., Araki, S., Block, W. D., Cohen, A. S., Jackson, C. E., Kuroiwa, Y., McKusick, V. A. G., Nissim, J., Sohar, E., and Van Allen, M. W. (1970). Hereditary amyloidosis. Arthritis and Rheumatism, 13, 902-915.

Barlow, J. B., Bosman, C. K., Pocock, W. A., and Marchand, P. (1968). Late systolic murmur and non-ejection ('midlate') systolic clicks. British Heart fournal, 30, 203-218.

Benchimol, A. (1977). Non-invasive Diagnostic Techniques in Cardiology, pp. 251 and 362. Williams and Wilkins, Baltimore.

Buja, L. M., Khoi, N. B., and Roberts, W. C. (1970). Clinically significant cardiac amyloidosis. Clinicopathologic findings in 15 patients. American fournal of Cardiology, 26, 394-405.

Coelho, E., and Pimentel, J. C. (1961). Cardiac involvement in a peculiar form of paramyloidosis. American fournal of Cardiology, 8, 624-632.

Frederiksen, T., Gøtzsche, H., Harboe, N., Kiaer, W., and Mellemgaard, K. (1962). Familial primary amyloidosis with severe amyloid heart disease. American fournal of Medicine, 33, 328-348.

Sawayama, T., Tohara, M., Katsume, H., and Nezuo, S. (1973). Polygraphic studies of the effect of nitroglycerin in patients with ischaemic heart disease. British Heart fournal, 35, 1234-1239.

Weissler, A. M., Harris, W. S., and Schoenfeld, C. D. (1968). Systolic time intervals in heart failure in man. Circulation, 37, 149-159.

Requests for reprints to Dr T. Sawayama, Department of Medicine, Kawasaki Medical School, Kurashiki, Japan 701-01. 